\title{
Ground state energy of the two-dimensional weakly interacting Bose gas: First correction beyond Bogoliubov theory
}

\author{
Christophe Mora ${ }^{1}$ and Yvan Castin ${ }^{2}$ \\ Laboratoire Pierre Aigrain ${ }^{1}$ and Laboratoire Kastler Brossel ${ }^{2}$, École Normale Supérieure and CNRS, \\ Université Denis Diderot $7^{1}$ and UPMC $C^{1,2}, 24$ rue Lhomond, 75005 Paris, France
}

(Dated: October 26, 2018)

\begin{abstract}
We consider the grand potential $\Omega$ of a two-dimensional weakly interacting homogeneous Bose gas at zero temperature. Building on a number-conserving Bogoliubov method for a lattice model in the grand canonical ensemble, we calculate the next order term as compared to the Bogoliubov prediction, in a systematic expansion of $\Omega$ in powers of the parameter measuring the weakness of the interaction. Our prediction is in very good agreement with recent Monte Carlo calculations.
\end{abstract}

PACS numbers: 05.30.Jp,03.75.Hh

Recent experimental progress with ultracold atoms has renewed the interest in the two-dimensional weakly interacting Bose gas [1, 2. In view of a comparison to future experimental results on the equation of state of the gas at low temperatures, this raises the question of the accuracy of existing theoretical work 3 , 4. Since the pioneering works of Schick [5] and Popov [6] on the energy of the weakly interacting Bose gas in two dimensions, several recent predictions have been obtained. In mathematical physics, it was proved that Schick's formula for the ground state energy is asymptotically exact in the limit of vanishing density [7. Numerically, very precise Monte Carlo calculations of the ground state energy have been performed 8, 9. Analytically, Popov's result was confirmed by a Bogoliubov type theory [10] (and by Monte Carlo calculations [9]) but several attempts to calculate analytically the energy beyond Popov's result have led to non-identical predictions [11, 12, 13.

The most systematic among the theoretical approaches are those relying on an expansion of the energy in powers of a small parameter. This is the case of the approaches [6, 10, which have led to the equation of state [14]

$$
\rho \simeq \frac{m \mu}{4 \pi \hbar^{2}} \ln \left(\frac{4 \hbar^{2}}{m \mu a^{2} e^{2 \gamma+1}}\right)
$$

where $m$ is the mass of a boson, $a>0$ is the twodimensional scattering length among the particles, $\mu$ is the chemical potential and $\gamma=0.57721566 \ldots$ is Euler's constant. Remarkably Eq.(1) is universal, i.e. it depends on the interaction potential through the scattering length only. One obtains from (1) the grand potential $\Omega=E-\mu N$ in the thermodynamic limit, where $E$ is the gas energy and $N$ the atom number, by a simple integration over $\mu$ since $N=-\partial_{\mu} \Omega$ :

$$
L^{-2} \Omega(\mu) \simeq-\frac{m \mu^{2}}{8 \pi \hbar^{2}}\left[\ln \left(\frac{4 \hbar^{2}}{m \mu a^{2} e^{2 \gamma+1}}\right)+\frac{1}{2}\right]
$$

where $L^{2}$ is the surface of the gas. The small parameter

$$
\epsilon(\mu)=\frac{1}{\ln \left[4 \hbar^{2} /\left(\mu m a^{2} e^{2 \gamma+1}\right)\right]}
$$

is apparent in (2).

In the vanishing density (or chemical potential) limit, the prediction (1) can be checked to be asymptotically equivalent to Schick's formula, as it should be. In a further expansion of the energy in terms of the density, the precise value of the constant under the logarithm in (1) eventually matters. In particular, a careful account of the low-energy two body $T$-matrix is essential to derive this constant [14]. Its value turns out to agree with recent Monte Carlo results [9, it however differs from the prediction of Ref. 12]. For not extremely small values of the density, a significant deviation is observed between the energy deduced from Eq. (2) and Monte Carlo results 8, 9, see in Fig.1 the fact that the symbols significantly deviate from unity. Furthermore, this deviation is not accounted for by the beyond-Bogoliubov theories of Refs. 11, 13, see in Fig 1 the fact that the symbols significantly deviate from the dashed and dotted lines.

In the present work we extend the Bogoliubov method [10] as in [15] and we go one step further than Eq. (2) in the expansion of the grand potential in powers of $\epsilon(\mu)$. We obtain in the thermodynamic limit:

$$
L^{-2} \Omega(\mu)=-\frac{m \mu^{2}}{8 \pi \hbar^{2}}\left[\frac{1}{\epsilon(\mu)}+\frac{1}{2}+\frac{8 I}{\pi} \epsilon(\mu)+\ldots\right],
$$

where the numerical constant $I$ is given by a multiple integral that we have evaluated numerically:

$$
I \simeq 1.0005 \ldots
$$

Since the extra term that we have found with respect to (2) is indeed $o(1)$, this analytically confirms that the numerical constant inside the logarithm in (1) is the correct one. Furthermore, the inclusion of the extra term leads to a now satisfactory agreement with the numerical results of [8, 9], see in Fig 1 the agreement of the solid line with the plotting symbols.

Our model: In a first stage we still consider a general value $d$ of the space dimension. As a regularization scheme to treat ultraviolet divergences, we use a lattice model [10] to represent the interacting Bose gas, with the 


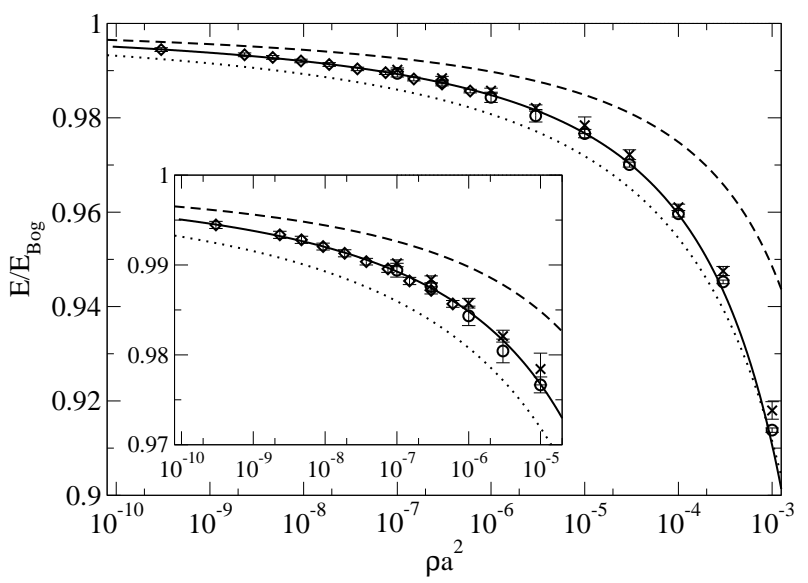

FIG. 1: Ground state energy $E$ of a two dimensional Bose gas, as a function of the gas density, and in units of the Bogoliubov prediction $E_{\text {Bog }}$ resulting from (2). Solid line: energy obtained from the beyond Bogoliubov analytical prediction (4) derived in this work. Dashed line: analytical prediction of [1] taking the exact expression $f(u)=u+u^{2} / 2+$ $2 u^{2} e^{2 / u} \operatorname{Ei}(-2 / u)$ of the function $f$ introduced in [11, where $\mathrm{Ei}$ is the function exponential integral. Dotted line: analytical prediction of [13] in the form 16]. Plotting symbols with error bars: numerical results of [8], for interactions given by hard disks (crosses) and by soft disks (circles); numerical results of 9], for dipolar interactions (diamonds). We have restricted the results of 8 ] to their universal range, that is to low enough values of $\rho a^{2}$ such that the hard disks and soft disks models give the same values of the energy within the error bars. For the same reason, we have restricted the results of [9] to $\rho a^{2}<10^{-6}$. The inset is a magnification.

grand canonical Hamiltonian

$$
H=\sum_{\mathbf{r}} \ell^{d} \hat{\psi}^{\dagger}\left[-\frac{\hbar^{2}}{2 m} \Delta-\mu\right] \hat{\psi}+\frac{g_{0}}{2} \sum_{\mathbf{r}} \ell^{d} \hat{\psi}^{\dagger} \hat{\psi}^{\dagger} \hat{\psi} \hat{\psi}
$$

where the limit $\ell \rightarrow 0$ is eventually taken to recover the continuous gas. The discrete positions $\mathbf{r}$ run over the lattice $\ell \mathbb{Z}^{d}$, where $\ell$ is the lattice spacing; there is no trapping potential, but a quantization box $[0, L]^{d}$ with periodic boundary conditions. The bosonic field obeys the discrete commutation relations $\left[\hat{\psi}\left(\mathbf{r}_{1}\right), \hat{\psi}^{\dagger}\left(\mathbf{r}_{2}\right)\right]=$ $\delta_{\mathbf{r}_{1}, \mathbf{r}_{2}} / \ell^{d}$. In the kinetic energy operator, $\Delta$ is a discrete representation of the Laplacian on the lattice, such that the plane wave $e^{i \mathbf{k} \cdot \mathbf{r}}$ is an eigenstate of $\Delta$ with the eigenvalue $-k^{2}$, the discrete nature of the lattice allowing one to restrict the values of $\mathbf{k}$ to the first Brillouin zone

$$
\mathcal{D}=[-\pi / \ell, \pi / \ell)^{d} .
$$

The on-site interactions are characterized by a coupling constant $g_{0}$ adjusted to reproduce the correct value of the scattering length $a$ in the two-body scattering problem, as detailed in [17. For the two-dimensional case one obtains

$$
g_{0}=\frac{2 \pi \hbar^{2}}{m} \frac{1}{\ln \left(K_{2} \ell / a\right)} \quad \text { with } \quad K_{2}=\frac{e^{-\gamma+2 G / \pi}}{\pi} .
$$

We shall find in $2 \mathrm{D}$ that the first correction beyond 2 is universal, it depends on the interaction only through the scattering length $a$ in the zero chemical potential limit. The microscopic details of our model, the fact that it is a lattice model or that Catalan's constant $G=0.91596 \ldots$ appears in (8), are thus not relevant in this limit.

Elimination of the condensate mode: We now assume that the ground state of $H$ in the thermodynamic limit is a condensate, so that we take $d=2[5,6$, or $d=3$. We then use Bogoliubov method to eliminate the condensate mode and obtain a Hamiltonian for the field of non-condensed particles. We use here a $U(1)$ symmetry preserving approach in the spirit of [18, 19], adjusted to the case of the grand canonical ensemble. We split the field operator as the sum of the condensate field and the field of the non-condensed modes:

$$
\hat{\psi}(\mathbf{r})=\phi(\mathbf{r}) \hat{a}_{\mathbf{0}}+\hat{\psi}_{\mathrm{nc}}(\mathbf{r}),
$$

where $\hat{a}_{\mathbf{0}}$ is the annihilation operator in the condensate mode $\phi(\mathbf{r})=1 / L^{d / 2}$. We eliminate the condensate particle number $\hat{n}_{\mathbf{k}=\mathbf{0}}=\hat{a}_{\mathbf{0}}^{\dagger} \hat{a}_{\mathbf{0}}$ using

$$
\hat{n}_{\mathbf{k}=\mathbf{0}}=\hat{N}-\hat{N}_{\mathrm{nc}}
$$

where $\hat{N}$ is the total number of particles and

$$
\hat{N}_{\mathrm{nc}}=\sum_{\mathbf{r}} \ell^{d} \hat{\psi}_{\mathrm{nc}}^{\dagger} \hat{\psi}_{\mathrm{nc}}
$$

is the number of non-condensed particles. To complete the elimination of the condensate mode, we introduce the representation 20]

$$
\hat{a}_{\mathbf{0}}=\hat{A} \hat{n}_{\mathbf{k}=\mathbf{0}}^{1 / 2} \quad \text { with } \quad \hat{A} \equiv\left(1+\hat{n}_{\mathbf{k}=\mathbf{0}}\right)^{-1 / 2} \hat{a}_{\mathbf{0}} .
$$

As shown in Eq.(5.40) of [20] one has the exact relations $\hat{A} \hat{A}^{\dagger}=1$ and $\hat{A}^{\dagger} \hat{A}=1-\left|\operatorname{vac}_{\mathbf{0}}\right\rangle\left\langle\operatorname{vac}_{\mathbf{0}}\right|$, where $\left|\operatorname{vac}_{\mathbf{0}}\right\rangle$ is the vacuum state for the condensate mode. The condensate mode elimination is completed by inclusion of $\hat{A}^{\dagger}$ in the non-condensed field, defining as in 19 the field operator

$$
\hat{\Lambda}(\mathbf{r})=\hat{A}^{\dagger} \hat{\psi}_{\mathrm{nc}}(\mathbf{r}),
$$

which conserves the total particle number. In the thermodynamic limit, the condensate mode has a vanishing probability to be empty, so that $\hat{A}^{\dagger} \hat{A} \rightarrow \hat{A} \hat{A}^{\dagger}=1$, and $\hat{\Lambda}$ and $\hat{\Lambda}^{\dagger}$ obey simple commutation relations in this limit.

In the canonical ensemble, it remains to inject the splitting (9) into $H$ and to eliminate the condensate mode, finally replacing the operator $\hat{N}$ by its known value $N$. One obtains contributions of various degrees in $\hat{\Lambda}$, starting from degree two. The terms of degree two in $\hat{\Lambda}$ gives the Bogoliubov Hamiltonian, the terms of higher degrees may be treated by perturbation theory.

In the grand canonical ensemble, however, the chemical potential $\mu$ rather than the particle number is known; at zero temperature, $\hat{N}$ does not fluctuate but assumes 
an a priori unknown value $N(\mu)$, a function of $\mu$ to be determined order by order in the weakly interacting limit. To zeroth order in $\hat{\Lambda}$, the gas is a pure condensate and one obtains the mean-field type relation

$$
N^{(0)}(\mu)=\frac{\mu L^{d}}{g_{0}} .
$$

It is then convenient to split $N$ as

$$
N(\mu)=N^{(0)}(\mu)+\delta N(\mu) .
$$

As we shall see, $\delta N(\mu)$ to leading order is second order in $\hat{\Lambda}$, as the mean number of non-condensed particles $\left\langle\hat{N}_{\mathrm{nc}}\right\rangle$.

After some calculation, neglecting unity as compared to the condensate atom number and replacing $\hat{N}$ with $N(\mu)$, we obtain the desired rewriting of the Hamiltonian with no reference to the condensate mode:

$$
\begin{aligned}
H & \simeq-\frac{1}{2} \mu N^{(0)}(\mu) \\
& +\sum_{\mathbf{r}} \ell^{d}\left[\hat{\Lambda}^{\dagger}\left(-\frac{\hbar^{2}}{2 m} \Delta\right) \hat{\Lambda}+\mu \hat{\Lambda}^{\dagger} \hat{\Lambda}+\frac{\mu}{2}\left(\hat{\Lambda}^{2}+\hat{\Lambda}^{\dagger 2}\right)\right] \\
& +\frac{g_{0}}{L^{d / 2}} \sum_{\mathbf{r}} \ell^{d}\left\{\left[N(\mu)-\hat{N}_{\mathrm{nc}}\right]^{1 / 2} \hat{\Lambda}^{\dagger} \hat{\Lambda}^{2}+\text { h.c. }\right\} \\
& +\frac{g_{0}}{2 L^{d}}\left\{\left[\delta N(\mu)+\hat{N}_{\mathrm{nc}}\right]^{2}-4 \hat{N}_{\mathrm{nc}}^{2}+\left[\delta N(\mu)-\hat{N}_{\mathrm{nc}}\right] \hat{X}\right. \\
& \left.+\hat{X}^{\dagger}\left[\delta N(\mu)-\hat{N}_{\mathrm{nc}}\right]\right\}+\frac{g_{0}}{2} \sum_{\mathbf{r}} \ell^{d} \hat{\Lambda}^{\dagger} \hat{\Lambda}^{\dagger} \hat{\Lambda} \hat{\Lambda} .
\end{aligned}
$$

We have used the fact that, in the spatially homogeneous case, one exactly has $\sum_{\mathbf{r}} \ell^{d} \hat{\Lambda}(\mathbf{r})=0$, and we have set

$$
\hat{X}=\sum_{\mathbf{r}} \ell^{d} \hat{\Lambda}^{2} .
$$

Perturbative expansion: We now expand (16) in powers of $\hat{\Lambda}$. Keeping terms up to second order in $\widehat{\Lambda}$ we obtain

$$
\begin{aligned}
H_{\leq 2}=-\frac{1}{2} \mu N^{(0)}(\mu) & +\sum_{\mathbf{r}} \ell^{d}\left[\hat{\Lambda}^{\dagger}\left(-\frac{\hbar^{2}}{2 m} \Delta\right) \hat{\Lambda}\right. \\
& \left.+\mu \hat{\Lambda}^{\dagger} \hat{\Lambda}+\frac{\mu}{2}\left(\hat{\Lambda}^{2}+\hat{\Lambda}^{\dagger 2}\right)\right] .
\end{aligned}
$$

This plays the role of the Bogoliubov Hamiltonian in the usual theory. Its ground state energy we thus call the Bogoliubov approximation for the grand potential:

$$
\Omega_{\mathrm{Bog}}(\mu)=-\frac{\mu^{2} L^{d}}{2 g_{0}}-\sum_{\mathbf{k} \in \mathcal{D}^{*}} \epsilon_{k} V_{k}^{2}
$$

where we have replaced $N^{(0)}(\mu)$ by its value and we have introduced the Bogoliubov modal amplitudes obeying

$$
U_{k}+V_{k}=\frac{1}{U_{k}-V_{k}}=\left(\frac{\hbar^{2} k^{2} /(2 m)}{2 \mu+\hbar^{2} k^{2} /(2 m)}\right)^{1 / 4} \equiv s_{k}
$$

and the corresponding Bogoliubov energies

$$
\epsilon_{k}=\left[\frac{\hbar^{2} k^{2}}{2 m}\left(\frac{\hbar^{2} k^{2}}{2 m}+2 \mu\right)\right]^{1 / 2} .
$$

Taking minus the derivative of 19 with respect to $\mu$ to obtain the atom number, and using

$$
\partial_{\mu}\left(\epsilon_{k} V_{k}^{2}\right)=-V_{k}\left(U_{k}+V_{k}\right),
$$

one recovers, in the thermodynamic limit, Eq.(152) in [10], and thus (1) in the limit $\ell \rightarrow 0$ [22].

To go beyond Bogoliubov, we collect into $H_{3}$ the terms of degree three in $\hat{\Lambda}$ and into $H_{4}$ the terms of degree four in $\hat{\Lambda}$, keeping in mind that $\delta N(\mu)$ is to leading order of degree two, $\delta N(\mu)=N^{(2)}(\mu)+\ldots$, so that

$$
\begin{aligned}
H_{3} & =g_{0}\left[\frac{N^{(0)}(\mu)}{L^{d}}\right]^{1 / 2} \sum_{\mathbf{r}} \ell^{d} \hat{\Lambda}^{\dagger}\left(\hat{\Lambda}+\hat{\Lambda}^{\dagger}\right) \hat{\Lambda} \\
H_{4} & =\frac{g_{0}}{2 L^{d}}\left\{\left[N^{(2)}(\mu)+\hat{N}_{\mathrm{nc}}\right]^{2}-4 \hat{N}_{\mathrm{nc}}^{2}+\left[N^{(2)}(\mu)-\hat{N}_{\mathrm{nc}}\right] \hat{X}\right. \\
& \left.+\hat{X}^{\dagger}\left[N^{(2)}(\mu)-\hat{N}_{\mathrm{nc}}\right]\right\}+\frac{g_{0}}{2} \sum_{\mathbf{r}} \ell^{d} \hat{\Lambda}^{\dagger} \hat{\Lambda}^{\dagger} \hat{\Lambda} \hat{\Lambda} .
\end{aligned}
$$

We treat $H_{4}$ to first order in perturbation theory and $\mathrm{H}_{3}$ to second order, to obtain the first correction to the Bogoliubov prediction for the grand potential:

$$
\delta \Omega=\left\langle H_{4}\right\rangle+\left\langle H_{3} \frac{1}{\Omega_{\mathrm{Bog}}-H_{\leq 2}} H_{3}\right\rangle
$$

where the expectation value is taken in the ground state of $H_{\leq 2}$, that is in the vacuum of the operators $\hat{b}_{\mathbf{k}}$ appearing in the modal expansion

$$
\hat{\Lambda}(\mathbf{r})=L^{-d / 2} \sum_{\mathbf{k} \in \mathcal{D}^{*}} \hat{b}_{\mathbf{k}} U_{k} e^{i \mathbf{k} \cdot \mathbf{r}}+\hat{b}_{\mathbf{k}}^{\dagger} V_{k} e^{-i \mathbf{k} \cdot \mathbf{r}} .
$$

To find the value of $N^{(2)}(\mu)$, we minimize $\left\langle H_{4}\right\rangle$ over $N^{(2)}$, keeping in mind that $H_{\leq 2}$ and $H_{3}$ do not depend on $N^{(2)}$. In the thermodynamic limit, one has $\left\langle\hat{N}_{\mathrm{nc}}^{2}\right\rangle \simeq\left\langle\hat{N}_{\mathrm{nc}}\right\rangle^{2}$ and $\left\langle\hat{N}_{\mathrm{nc}} \hat{X}\right\rangle \simeq\left\langle\hat{N}_{\mathrm{nc}}\right\rangle\langle\hat{X}\rangle$, so that

$$
N^{(2)}(\mu) \simeq-\left\langle\hat{N}_{\mathrm{nc}}+\frac{\hat{X}+\hat{X}^{\dagger}}{2}\right\rangle=-\sum_{\mathbf{k} \in \mathcal{D}^{*}} V_{k}\left(U_{k}+V_{k}\right) .
$$

The divergence of $N^{(0)}(\mu)$ when $\ell \rightarrow 0$ is removed in the combination $N^{(0)}(\mu)+N^{(2)}(\mu)$. The resulting density is in agreement with Eq. (1). One is then left with

$$
\left\langle H_{4}\right\rangle \simeq-\frac{g_{0}}{L^{d}}\left\langle\hat{N}_{\mathrm{nc}}\right\rangle\left[\left\langle\hat{N}_{\mathrm{nc}}\right\rangle+2\langle\hat{X}\rangle\right] .
$$

Using the modal expansion (26) and Wick's theorem we finally obtain after some calculation

$$
\begin{aligned}
& \delta \Omega \simeq-\frac{\mu^{2}}{N^{(0)}(\mu)+N^{(2)}(\mu)} \sum_{\mathbf{k}_{1}, \mathbf{k}_{3} \in \mathcal{D}^{*}}\left\{\frac{V_{3}^{2} V_{1}\left(U_{1}+s_{1}\right)}{\mu}\right. \\
& \left.+\left(1-\delta_{\mathbf{k}_{2}, \mathbf{0}}\right) \frac{U_{1} s_{2} V_{3}}{\epsilon_{1}+\epsilon_{2}+\epsilon_{3}} \sum_{\sigma \in S_{3}} U_{\sigma(1)} s_{\sigma(2)} V_{\sigma(3)}\right\} .
\end{aligned}
$$


We have introduced the vector $\mathbf{k}_{2} \in \mathcal{D}$ such that $\mathbf{k}_{1}+$ $\mathbf{k}_{2}+\mathbf{k}_{3} \in(2 \pi / l) \mathbb{Z}^{d}$. The notation $U_{i}, i \in\{1,2,3\}$, stands for $U_{k_{i}}$. The sum over $\sigma$ runs over the permutation group $S_{3}$. For convenience we have added $N^{(2)}(\mu)$ to $N^{(0)}(\mu)$ in the denominator of the overall factor in $(29)$, which is allowed at the present order of the calculation.

Absence of divergences in 2D: We now take the thermodynamic limit, replacing sums over $\mathbf{k}$ by integrals over the domain $\mathcal{D}$ in 29 . We also take the zero lattice spacing limit $\ell \rightarrow 0$ [22] so that the integration domain over $\mathbf{k}$ is now $\mathbb{R}^{d}$. Since $\mathbf{k}_{2}=-\left(\mathbf{k}_{1}+\mathbf{k}_{3}\right)$ and the integrand depends only on the moduli $k_{1}, k_{2}$ and $k_{3}$, see $(29)$, we are left with a triple integral over $k_{1}, k_{3}$ and the angle between the vectors $\mathbf{k}_{1}$ and $\mathbf{k}_{3}$. In $2 \mathrm{D}$, we show below that this integral converges, that is it has neither an infrared nor an ultraviolet divergence. The first correction beyond the Bogoliubov energy is thus universal in 2D. Since convergence is established, we can resort to numerical integration. After the change of variables $q_{i}=\hbar k_{i} /(2 m \mu)^{1 / 2}$ and pulling out a factor $\pi\left[(2 m \mu)^{1 / 2} L /(2 \pi \hbar)\right]^{4} / \mu$, we get (5). Summing $\Omega_{\mathrm{Bog}}$ to $\delta \Omega$ we then obtain (4).

To show the infrared convergence, we replace the integrand by its leading low- $k_{i}$ behavior: $U_{i}$ and $V_{i}$ diverge as $1 / \sqrt{k_{i}}, s_{i}$ vanishes as $\sqrt{k_{i}}$ and $\epsilon_{i}$ vanishes as $k_{i}$. Including the Jacobian factors $k_{1}$ and $k_{3}$ from $2 \mathrm{D}$ integration in polar coordinates, we see that the integral of the first term in the curly brackets of (29) converges. The contribution of the term due to permutation $\sigma$ scales as

$$
\frac{k_{1} k_{3}}{k_{1}+k_{2}+k_{3}}\left(\frac{k_{2}}{k_{1} k_{3}}\right)^{1 / 2}\left(\frac{k_{\sigma(2)}}{k_{\sigma(1)} k_{\sigma(3)}}\right)^{1 / 2}<1,
$$

so its integral over $k_{1}$ and $k_{3}$ is also convergent.

For the ultraviolet convergence, the full reasoning is rather long [21, so we give a simplified explanation. We approximate each term in the integrand in 29 by its leading high- $k_{i}$ behavior, $U_{i}$ and $s_{i}$ tending to unity, $V_{i}$ vanishing as $1 / k_{i}^{2}$ and $\epsilon_{i}$ diverging as $k_{i}^{2}$. In the sum over $\sigma$, the terms with $\sigma(3) \neq 3$ are not dangerous. E.g. for $\sigma(3)=1$, a factor $V_{1} V_{3}$ appears, and including the Jacobian factors, one obtains a contribution scaling as

$$
\frac{k_{1} k_{3}}{k_{1}^{2}+k_{2}^{2}+k_{3}^{2}} \times \frac{1}{k_{1}^{2} k_{3}^{2}}<\frac{1}{2 k_{1}^{2} k_{3}^{2}},
$$

so that the resulting double integral over $k_{1}$ and $k_{3}$ is convergent at infinity. The dangerous terms in the sum over $\sigma$ thus correspond to $\sigma(3)=3$ : the factor $V_{3}^{2}$ ensures convergence of the integral over $\mathbf{k}_{3}$ over a $\mathbf{k}_{1}$-independent range $\sim\left(m \mu / \hbar^{2}\right)^{1 / 2}$. At large $\mathbf{k}_{1}$, the energy denominator $\epsilon_{1}+\epsilon_{2}+\epsilon_{3}$ approaches $2 \epsilon_{1}$. Then, from the asymptotic relation $V_{1} \simeq-\mu /\left(2 \epsilon_{1}\right)$, we see that the two dangerous contributions coming from the permutations with $\sigma(3)=3$ exactly compensate with the first term $\simeq 2 V_{3}^{2} V_{1} / \mu$ in the curly brackets of 29 , which avoids an ultraviolet divergence of $\delta \Omega$.

In conclusion, we have calculated analytically and in a systematic way the first correction to the Bogoliubov prediction for the ground state grand potential of a $2 \mathrm{D}$ weakly interacting Bose gas. We find that this correction is universal, depending on the interaction potential through the scattering length only. It allows to describe analytically the not extremely weakly interacting regime, and contrarily to other analytical works, we obtain a prediction for the ground state energy in excellent agreement with the numerical results of [8, 9] over the range where the results of [8, 9] are model independent.

This work was stimulated by discussions with Elliot Lieb and Jakob Yngvason. The group of Y.C. is a member of IFRAF.

[1] Z. Hadzibabic et al., Nature 441, 1118 (2006).

[2] P. Cladé et al., arXiv:0805.3519 (2008).

[3] A. Posazhennikova, Rev. Mod. Phys. 78, 1111 (2006).

[4] I. Bloch, J. Dalibard, and W. Zwerger, Rev. Mod. Phys. 80, 885 (2008).

[5] M. Schick, Phys. Rev. A 3, 1067 (1971).

[6] V.N. Popov, Theor. Math. Phys. 11, 565 (1972).

[7] E. Lieb and J. Yngvason, J. Stat. Phys. 103, 509 (2001).

[8] S. Pilati, J. Boronat, J. Casulleras and S. Giorgini, Phys. Rev. A 71, 023605 (2005).

[9] G.E. Astrakharchik et al., arXiv:0812.3844 1 (2008).

[10] C. Mora and Y. Castin, Phys. Rev. A 67, 053615 (2003).

[11] A.Yu. Cherny and A.A. Shanenko, Phys. Rev. E 64, 027105 (2001).

[12] J.O. Andersen, Eur. Phys. J. B 28, 389 (2002).

[13] L. Pricoupenko, Phys. Rev. A 70, 013601 (2004).

[14] We found that the quantity $\epsilon_{0}$ appearing in Popov's theory is simply $\epsilon_{0}=4 \hbar^{2} /\left(m a^{2} e^{2 \gamma}\right)$. This is obtained by comparing the low- $k$ expression of the two-body $T$ matrix in Eq.(3.2) of [6] with Eq.(160) of [10].

[15] T. T. Wu, Phys. Rev. 115, 1390 (1959).

[16] We used the relations $m \mu a^{2} / \hbar^{2}=t^{2}[1-1 / \ln (q t)]$ and $2 \pi \rho a^{2}=t^{2}[-\ln (q t)+1 / 2]$ with $q=\exp (\gamma) / 2$ and $0<$ $t<1 / q$ (L. Pricoupenko, private communication).

[17] Y. Castin, J. Phys. IV (France) 116, 89 (2004).

[18] C.W. Gardiner, Phys. Rev. A 56, 1414 (1997).

[19] Y. Castin, R. Dum, Phys. Rev. A 57, 3008 (1998).

[20] P. Carruthers, M. Nieto, Rev. Mod. Phys. 40, 411 (1968).

[21] Setting $q_{i}=\hbar k_{i} /(2 m \mu)^{1 / 2}$, we used e.g. $\left|2 s_{2} V_{2}\right|=\mid s_{2}^{2}-$ $1 \mid \leq 2 /\left(2+q_{2}^{2}\right)$ and $\left(\epsilon_{1}-\epsilon_{2}\right) /\left(q_{1}^{2}-q_{2}^{2}\right) \leq 1+2 /\left(q_{1}^{2}+q_{2}^{2}\right)$.

[22] More precisely, in 2D, our expansion in powers of $\hat{\Lambda}$ is an expansion in powers of $\epsilon(\mu)$ for a fixed value of $\eta(\mu)=\ln (\xi / \ell) \gg 1$, where $\hbar^{2} /\left(m \xi^{2}\right)=\mu$. The Bogoliubov method indeed relies on a Born expansion 23] (here in powers of $g_{0}$ ) of the scattering amplitude $f_{k}$ for $k \simeq$ $1 / \xi$. From Eq.(167) of [10] the small parameter for the Born expansion is $2 \eta \epsilon$. This small parameter is explicitly obtained in our approach, from the requirement $N^{(2)} \ll$ $N^{(0)}$ : Estimating from 27 $N^{(2)} \simeq \eta L^{2} m \mu /\left(2 \pi \hbar^{2}\right)$ for $\ell \ll \xi$, we get $N^{(2)} / N^{(0)} \simeq 2 \eta \epsilon /(1+2 \eta \epsilon)$. One then takes the limit $\eta \rightarrow+\infty$ in each coefficient of the expansion of $\Omega$ in powers of $\epsilon$. This limit is exponentially fast approached in $\eta$ : we find that $\eta=7$ is more than large enough, it gives the value of $I$ in $\sqrt{5}$ at the $10^{-5}$ level.

[23] E.H. Lieb and W. Liniger, Phys. Rev. 130, 1605 (1963). 